\title{
EQUIVALENCE, CULTURE ET TRADUCTION
}

De nos jours, la connaissance de deux langues ne peut plus suffire pour bien traduire, étant donné que les différences entre les termes réputés comme équivalents ne se situent pas simplement au niveau de leurs signifiés dont les aires sémantiques ne sont pas superposables.

On le sait, et ce depuis Saussure, que les mots ne sont pas chargés "de présenter des concepts donnés d'avance"1. Et par conséquence les termes qu'on croit intuitivement équivalents, ne le sont, en fait, que partiellement.

Consulter les dictionnaires bilingues franco-arabes dont les carences se voient d'une façon évidente, n'est pas d'un grand secours, car le lexique dans la plupart de ces dictionnaires se présente souvent sous forme de mots isolés, et les articles ne sont pas suffisamment exemplifiés.

Or, pour faire face aux difficultés surgies au cours du processus de la traduction, nous estimons nécessaire de procéder à des analyses linguistiques approfondies et à des comparaisons différentielles permettant de délimiter le degré d'équivalence entre les termes français et leurs dits équivalents en arabe.

En effet, bien traduire un texte suppose des connaissances approfondies des faits linguistiques que présentent la langue source et la langue cible notamment ceux qui concernent de près le problème d'équivalences.

Dans ce but, et pour donner une idée précise de la façon dont se pose le problème d'équivalence lors du passage d'une langue à l'autre, nous nous proposons d'explorer les différents problèmes touchant, en particulier aux différents niveaux de langue (lexical, morphologique et syntaxique) et à la culture.

\section{MORPHOLOGIE ET TRADUCTION}

L'arabe se distingue du français par la flexion interne. La racine en arabe est une suite de consonnes généralement trilitère. Les voyelles en arabe ne sont que des éléments de dérivation ${ }^{2}$.

La racine consonantique, appelée "forme nue" par les anciens grammairiens arabes est liée à une notion déterminée. Ainsi la racine $k t b$ (kataba) exprime la notion d'“écrire", $q t l$ (qatala) exprime la notion de "tuer", etc. Selon les processus de la

1 Saussure F., Cours de linguistique générale, p. 161

2 Blachère et coll., Grammarie de l'arabe classique, pp. 36-76 
dérivation en arabe (alternance vocalique, gémination de la deuxième consonne, affixation) nous obtenons à partir de la racine consonantique de nombreuses formes verbales et nominales.

De toute évidence, ce qui nous intéresse ici est le dérivé (forme verbale ou nominale) en tant qu'unité lexicale douée d'un sens.

Sur le plan interlingual, il n'est pas toujours aisé de trouver un équivalent de ces dérivés.

Par exemple, le verbe arabe $d r b$ (daraba) peut être traduit en français par le verbe "battre", mais on ne trouve pas toujours d'équivalents sur le même niveau de structuration linguistique pour certains dérivés du verbe "daraba".

Ainsi "Tadaraba" (forme verbale préfixée par "ta", exprime en français le sens de "se combattre les uns contre les autres". "Darrab", (forme nominale obtenue par la gémination de la deuxième consonne) exprime en français le sens de "qui frappe beaucoup", etc.

On ne doute pas que les aires sémantiques des unités lexicales issues des procédés de formation tout à fait dissemblables, ne se recouvrent souvent que partiellement.

\section{CONSTRUCTIONS SYNTAXIQUES ET TRADUCTION}

Un terme isolé a de multiples valeurs potentielles, il s'avère donc très difficile d'établir une correspondance exacte entre les termes de deux langues (le français et l'arabe) hors contexte.

En effet le sens d'un terme se détermine par rapport à l'énoncé dans lequel il est susceptible d'apparaître. C'est-à-dire qu'il y a un lien entre le sens d'un terme et sa construction syntaxique.

Ainsi le verbe "donner" aura des sens différents selon qu'il sera employé dans les constructions: a) $\mathrm{N}$ donner $\mathrm{N}_{1}$ à $\mathrm{N}_{2}$, b) $\mathrm{N}$ donner sur $\mathrm{N}$, c) $\mathrm{N}$ donner dans $\mathrm{N}$, etc.

Sur le plan interlingual, le choix de l'équivalent convenable en arabe sera régi par le sens exprimé par le verbe "donner" dans ses différentes constructions.

"Donner" au sens d'"offrir" se traduit en arabe par (a'ta) dans la construction $\mathrm{N}$ donne $\mathrm{N}_{1}$ à $\mathrm{N}_{2}$.

Exemple:

- Il donne un livre à son ami

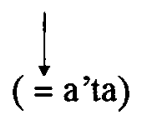

Dans la construction $\mathrm{N}$ donne sur $\mathrm{N}$, “donner" au sens de "d'où l'on voit" se traduit en arabe par le verbe "ašrafa". 


\section{Exemple:}

- La fenêtre donne sur la mer.<smiles>C[14CH3]</smiles>

Tandis que dans la construction $\mathrm{N}$ donne dans $\mathrm{N}$, "donner" au sens de "qui conduit à" se traduit en arabe par le verbe "yagoudou".

Exemple:

- Le couloir donne dans le garage.

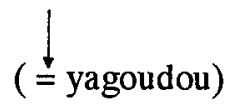

\section{CULTURE ET TRADUCTION}

Certaines valeurs qui s'ajoutent au signifié d'un terme sont appelées "significations culturelles." Ces significations concernent souvent la manière de se représenter le monde, le découpage de la réalité, etc.

Ainsi l'expression "poser un lapin" qui signifie "ne pas venir au rendez-vous qu'on a donné à quelqu'un". Cette expression présente une valeur spécifique à la langue française.

Cette signification, dite culturelle, bien qu'elle soit connue par les natifs français, risque de poser un problème de compréhension pour les arabophones, et ne peut se traduire au même niveau de structuration linguistique.

En effet, l'extension du sens du mot français "lapin" et celle de son équivalent arabe "arnab" n'est pas identique.

Il en va de même pour d'innombrables termes dont les aires sémantiques ne sont pas superposables lors du passage du français à l'arabe et vice-versa.

Il en découle que le traducteur ne peut ignorer la disparité de correspondances en particulier celles liées au contexte culturel - entre les mots français et leurs équivalents arabes.

\section{CONCLUSION:}

Cette étude démontre que les mots ne sont pas des unités isolés, et que le sens d'un mot dépend de l'ensemble de ses rapports avec ses co-occurrents sur l'axe syntagmatique, et ses corrélés sur l'axe paradigmatique.

Ainsi, très souvent, une similarité parfaite de sens ne peut s'établir entre les termes français et leurs dits équivalents en arabe. 
Par conséquent il incombe au traducteur de créer lui-même l'équivalence de sens contextuel lorsque celle-ci ne concorde pas avec l'équivalence de signification hors contexte.

\section{BIBLIOGRAPHIE}

Bastin G., "Traduire, adapter, réexprimer", Meta, vol. 35, No. 3, 1990.

Bejoint H., "Trois dictionnaires récents: lequel choisir?" Les langues modernes, Paris, 1978.

Blachère et coll., Grammaire de l'arabe classique. G. P. Maisonneuve et larose, France, 1981.

Borel M.J., “Objet et discours de représentation”, Langage, No. 103, 1991.

Darbelent J., “A propos de la stylistique comparée à l'enseigement de la traduction”, Meta, vol. 33, No. 2, 1988.

Delisle J., L'analyse du discours comme méthode de traduction, University of Ottawa press, Canada, 1980.

Dubois J., "Dictionnaire et syntaxe", Lexique No. 2, Presse universitaire de Lille, 1983.

Edwards J., "Literarcy and education in context of cultural and linguistic heterogeneity ", La Revue canadienne des langues vivantes, vol. 47, No. 5, 1991.

Fehri F., Linguistiqe arabe, forme et interprétation, Publication de la faculté de lettres et sciences humains, Maroc, 1982.

Fleish H., Traité de philologie arabe, vol. 2, Dar El-Mashreq, Liban, 1979.

Gak V.G., Lexicologie contrastive, relations internationales, Moscou, 1977.

Galisson R., Des mots pour communiquer, CLE international, France 1983.

Gentilhomme Y., Essai d'approche microsystémique, Peter Lang., Francfort's, Main New York, 1985.

Gilli Y., "A propos du texte littéraire et de F. Kafka” Annales littéraires de l'Université de Besançon, 1985.

Gross G., "Lexicographie et grammaire", Cahier de lexicologie, No. 39 Besançon, 1981.

Hagège Cl., La structure des langues, P. U. F. “Que sais-je?”, France, 1982.

Ibrahim A., "Neologisme par télescopage", le Français dans le monde, No. 182, France, 1984.

Laurian A. M., "Humour et traduction au contact des cultures", Meta, vol. 34, No. 1, 1989.

Lethuilier J., "La synonymie en langue de spécialité" Meta, vol. 34, No. 3, 1989.

Lomholt K., "Problems of intercultural translation", Babel, vol. 37, No. 1, 1991.

Mdibeh A., "Fertilisation ou intégration", Meta, vol. 32, No. 3, 1987. 
Mel'cuk I. et coll., Dictionnaire explicatif et combinatoire du français contemporain, Montréal, 1984.

Monteil V., L'arabe moderne, Klincksieck, France 1960.

Peytard J., "De la diffusion d'un élément préfixal: mini". Langue française, No. 17, 1973.

Ramat P., Typologie linguistique, P. U. F. France, 1985.

Rey-Debove J., “Un Dictionnaire morphologique?”, Le français aujourd'hui, No. 58, 1982.

Saussure F., Cours de linguistique générale, Payot, France 1976.

Tournier J., "Humour et lexique", Annales littéraires de l'Université de Besançon, 1981.

Povzetek

PREVAJANJE: JEZIKOVNA IN ZUNAJJEZIKOVNA USTREZNOST

Prispevek želi opozoriti na pasti, ki jih skriva prevajanje iz jezika v jezik, $v$ tem primeru iz francoščine $v$ arabščino in narobe. So dvojne narave: čisto jezikovne, pa tudi na kulturni ravni.

Izraze v jeziku, iz katerega se prevaja, je treba razumeti v širšem sobesedilu, na kulturni ravni jezika, in jih tako tudi prevajati, torej poiskati ustrezni izraz na kultumi ravni jezika, v katerega se prevaja. Zgolj intuitivna izbira izraza, ne da bi upoštevali zunajjezikovni okvir, prav lahko povzroči nesporazum. 